Revue d'histoire de l'Amérique française

ZAS REVUE D.HISTOIRE DE L'AMÉRIQUE FRANÇAISE

\title{
Le nationalisme canadien dans le domaine religieux
}

L'affaire de l'abbé Thavenet

\section{Wilfrid-H. Paradis}

Volume 7, numéro 4, mars 1954

URI : https://id.erudit.org/iderudit/301619ar

DOI : https://doi.org/10.7202/301619ar

Aller au sommaire du numéro

Éditeur(s)

Institut d'histoire de l'Amérique française

ISSN

0035-2357 (imprimé)

1492-1383 (numérique)

Découvrir la revue

Citer cet article

Paradis, W.-H. (1954). Le nationalisme canadien dans le domaine religieux :

l'affaire de l'abbé Thavenet. Revue d'histoire de l'Amérique française, 7(4),

465-482. https://doi.org/10.7202/301619ar d'utilisation que vous pouvez consulter en ligne.

https://apropos.erudit.org/fr/usagers/politique-dutilisation/ 


\title{
LE NATIONALISME CANADIEN DANS LE DOMAINE RELIGIEUX
}

\author{
L'AFFAIRE DE L'ABBE THAVENET *
}

\section{Introduction}

Devant le grand nombre de sociétés consacrées à développer l'amitié franco-canadienne tout comme à la vue des efforts faits de part et d'autre pour renouer les liens qui unissent la France au Canada, nous serions loin de nous douter qu'il n'y a pas très longtemps encore, une profonde antipathie divisait les deux peuples. Ce qui nous surprend davantage c'est que ce ressentiment ait envahi le domaine ecclésiastique pour opposer les prêtres venus de France à ceux de naissance canadienne.

Après l'abandon de la Nouvelle-France par Louis XV en 1763, le gouvernement anglais interdit le passage de prêtres français dans la colonie. A ce moment l'entente est plutôt relative entre ecclésiastiques français et canadiens. Mais, à partir du début du XIXe siècle, les relations devienrent plus tendues. Les Sulpiciens du Séminaire de Montréal insistent pour un recrutement de leur communauté en France et semblent se réserver les charges importantes de la maison de préférence aux Canadiens. Ceux-ci portent le gros de leurs attaques contre la compagnie de Saint-Sulpice. Y a-t-il d'autres motifs a cet antagonisme?

Nous ne pouvons élucider entièrement cette question. Elle comporte des éléments psychologiques qui dépassent les bornes d'une étude comme celle-ci. Le fond du probleme nous a paru être celui de la relève du clergé métropolitain par le clergé indigène. Cas qui n'est pas unique dans les annales des missions chrétiennes.

* Mémoire présenté au professeur Ch.-A. Julien, pour le Doctorat d'Université de l'Université de Paris. 
De plus, entre 1763 et 1800 , le Canadien est devenu conscient de sa nouvelle nationalité. Bien que de langue française, plus encore qu'au 18e siècle, la distinction s'accentue entre lui et le Français venu de France. Ajoutons à cela quelques rivalités personnelles, puis la détermination d'empêcher les grandes seigneuries du Séminaire de passer à la couronne britannique, lors de la disparition du dernier Sulpicien français. Et nous aurons tous les éléments d'une mésentente qui eut d'assez graves conséquences.

Nous avons choisi M. Thavenet, un Sulpicien, comme sujet de ce mémoire, parce qu'il a été, pour ainsi dire, le centre de tous les désaccords entre sa communauté et les ecclésiastiques canadiens entre 1794, l'année de son arrivée au Canada, et 1844, l'année de sa mort à Rome. De plus, une querelle personnelle s'éleva entre le Sulpicien et l'évêque de Québec sur la reddition des comptes de certaines rentes qui lui avaient été confiées. Querelle qui se prolongea de 1834 à 1844.

L'idée de ce mémoire nous est venue de M. Claude de Bonnault. En dépouillant les archives de la Compagnie de Saint-Sulpice à Paris, il avait amassé des notes sur le sujet. Séduit par l'intérêt et l'originalité du nationalisme canadien dans le domaine religieux, il nous a conseillé de l'étudier plus à fond. Grâce à l'aide de M. de Bonnault, nous avons pu retrouver toute la correspondance et tous les documents importants.

Ce nationalisme canadien, - nous employons cette expression faute de mieux - ne s'est pas exclusivement centré autour de l'abbé Jean-Baptiste Thavenet, Sulpicien français. Un tel sentiment couvait à l'évêché de Québec et dans les communautés religieuses du pays depuis plusieurs années. Monsieur Thavenet eut seulement le malheur de s'occuper d'affaires canadiennes à un moment où les relations entre sa congrégation et l'épiscopat du pays tendaient a se gâter. Comme ce Sulpicien n'etait pas homme à prendre à la légère les attaques dirigées contre son Institut et surtout contre lui-même, il a été, pendant plusieurs années, un des points de mire d'une lutte regrettable. Une affaire, très simple au début, allait se compliquer, s'embrouiller au fil des années. Peut-être plus que tout autre, M. Thavenet contribua-t-il à multiplier les difficultés entre les ecclésiastiques français et les nouveaux citoyens britanniques. A parler franc, il fut un partisan entêté, à un moment où il était 
rare de trouver un ecclésiastique, canadien ou français, prêt à travailler à une solution juste et équitable des problèmes qu'ils avaient à résoudre. C'est par où $M$. Thavenet prend de l'importance dans une étude sur le nationalisme canadien dans le domaine religieux.

\section{Ministère de l'abbé Thavenet au Canada}

M. Thavenet est né à Chateauroux, dans le diocèse de Bourges, le 2 septembre 1763, l'année où la France cède la Nouvelle-France à l'Angleterre ${ }^{1}$. Nul n'aurait pu prévoir que cette coincidence marquerait son destin. Après des études à Bourges, où il arrive le $1^{\text {er }}$ novembre 1782, il est ordonné prêtre le 11 avril $1789^{2}$. Encore une fois le destin semble le marquer pour le Canada. Les sentiments bien connus de M. Thavenet pendant la Révolution Française ne lui permettent pas de rester en France. Il lui faut partir. Mais depuis le traité de Paris, le gouvernement anglais interdit absolument l'admission de Sulpiciens français dans leur ancienne maison de Montréal ${ }^{3}$. Les autorités britanniques ne lèvent leur interdiction qu'à l'heure de la Révolution. Et c'est ainsi qu'en septembre 1794, M. Thavenet figure parmi les onze Sulpiciens qui débarquent à Montréal pour reprendre le travail de cette congrégation, languissante depuis la conquête du Canada par les Anglais.

Nous ne savons que très peu de chose sur cette période de la vie de $M$. Thavenet au Canada. Il ne prend de l'importance qu'après avoir quitté le pays. Nous savons tout au plus que, du $1^{\text {er }}$ septembre 1794 au mois d'Octobre 1815, date de son départ, il exerce un ministère bien modeste à la paroisse de Montréal et au Lac de la Montagne, mission indienne des Sulpiciens, où il séjourne de 1802 à 1809. Ensuite, il est envoyé au Collège de Montréal où il remplit plusieurs charges jusqu'à l'année de son départ, six ans après ${ }^{4}$. Pendant ces 21 ans de travail au Bas-Canada, l'abbé Thavenet ne s'adonne qu'à une seule chose qui lui vaudra une certaine notoriété

1. H. Gauthier, La Compagnie de Saint-Sulpice au Canada (1912), 268.

2. Ibidem.

3. Nous reviendrons sur ce sujet au cours de cette étude.

4. Gauthier, op. cit., 269. 
plus tard. Il apprend l'algonquin et prépare un dictionnaire et une grammaire de cette langue indienne. Au dire de ses confrères, il ne sait l'algonquin que très imparfaitement, et de telles connaissances ne sont pas très rares au Canada. Grâce à ce vernis linguistique, le sulpicien impressionse les autorités romaines parmi lesquelles il va vivre les dernières années de sa vie ${ }^{5}$.

N'allons pas penser, pout tout cela, que $M$. Thavenet manquait de talent et qu'il n'aurait su gagner l'estime de ses confrères, ni des évêques du Canada. En 1815 les Sulpiciens de Montréal lui manifestèrent leur confiance en le choisissant pour remplir une tâche à la fois difficile et délicate. Tâche qui devait le ramener en France et le plonger au centre des affaires canadiennes et de difficultés surgies depuis longtemps entre les Messieurs de Saint-Sulpice et l'épiscopat et le clergé du Canada.

Dans la récente guerre entre l'Angleterre et la France, le gouvernement français a confisqué, à titre de propriété anglaise, les rentes possédées en France par les établissements catholiques du Canada. A la paix de 1814, il s'engage à les restituer. Chargé de s'occuper de l'affaire, M. Thavenet rentre en France. Il a pour mission de réclamer les rentes de tous les établissements de Montréal ${ }^{6}$. En même temps, il s'occupe des intérêts des communautés et établissements de Québec qui ont également des rentes à revendiquer dans l'ancienne mère-patrie. Il s'agit de sommes considérables et de revendications dont le bien-fondé n'est pas toujours facile à établir. L'évêque de Québec, ainsi que les cinq communautés de cette ville qui lui confièrent leurs intérêts faisaient donc confiance à leur chargé d'affaires et, de même aux Sulpiciens en général ${ }^{7}$.

L'abbé consacre un certain nombre d'années à la recherche

5. Fonds Canadien du Seminaire de Saint-Sulpice: lettre de Mgr Lartigue d l'abbé Maguire à Rome, 1834. M. Thavenet écrira lui-même aux prêtres du Séminaire de Montréal: "Le Pape m'a chargé de traduire en algonquin le petit catéchismo de Bellarmin et des extraits de l'Ecriture Bainte...". "La Propagande veut faire imprimer ma grammaire et mon dictionnaire de la langue algonquine", Ibidem, to 18 janvier 1832.

6. Discussion de $M$. Thavenet avec Québec sur la reddition des comptes (18341844). Mon affaire avec Monseigneur l'Archevêque de Québec, au sujet de mes Comples avec les clablissements de son Diocèse (s.d.), 1. Imprimé.

\section{Ibidem.}


des titres des diverses institutions canadiennes. Il ne trouve ceux de l'évêché de Québec et des Ursulines qu'en 1823. D'après son propre témoignage, il met dix ans en tout au recouvrement des rentes, ce qu'il n'accomplit qu'au prix de peines incroyables, et sans honoraires, sars aucun émolument, sans aucune rétribution quelconque $^{8}$. Son dévouement rapporte près de deux millions de francs aux établissements du Diocèse de Québec ${ }^{9}$.

Si la gérance de $M$. Thavenet profitait aux communautés canadiennes elle ne se terminait pas rapidement. De fait, l'évêque de Québec était loin de croire que cet agent de Saint-Sulpice qui était aussi le sien, avait tiré le meilleur parti possible de la mission à lui confiée. Tout en reconnaissant les réels services que $M$. Thavenet avait rendus au diocèse ${ }^{10}$, l'évêque voulut s'occuper lui-même de ces affaires et somma l'abbé de lui rendre compte de sa gestion $\mathbf{M}$. Thavenet était alors à Rome. Monseigneur Panet avait envoyé dans la Ville Étercelle, un de ses prêtres, l'abbé Maguire, avec mission de solliciter la coadjutorerie de Québec, pour un certain abbé Turgeon. Il le chargea en même temps de tirer au clair les comptes de M. Thavenet avec les institutions religieuses de Québec ${ }^{11}$.

L'abbé Maguire et Monsieur Thavenet ne purent arriver à aucune conclusion satisfaisante. Trois mois de pourparlers à Rome, en 1834, ne firent qu'embrouiller davantage cette affaire. Le représentant de l'Évêque de Québec découvrit plusieurs erreurs de calcul et qu'affectaient un nombre considérable de francs et de livres. Il ne put accepter les comptes que voulut rendre l'abbé Thavenet ${ }^{12}$.

Nous ne voulons pas nous engager dans une étude approfondie des erreurs relevées par l'abbé Maguire dans les comptes du Sulpicien. Ce serait trop compliqué et la chose n'offre d'intérêt qu'à un

\section{Ibidem.}

9. Ibid., 2.

10. Voir Infra pour les marques de reconnaissance des évêques de Québec envers $M$. Thavenet.

11. Discussion de $M$. Thavenet... Mon affaire avec Monseigneur..., 3.

12. Ceci ressort des rapports de M. Maguire a Mgr Panet et de la propre correspondance de $\mathbf{M}$. Thavenet. Voir aussi Discussion de $\mathbf{M}$. Thavenet... Mon affaire avac Monseigneur..., op. cit., 2-3. 
expert comptable. Ce qui importe, c'est que les deux prêtres ne purent s'entendre. D'où la brèche élargie entre les Sulpiciens et l'évêque de Québec.

Mgr Panet n'accusa jamais M. Thavenet de détournement de fonds, non plus que d'agissements malhonnêtes à l'égard des communautés qui lui avaient confié leurs affaires. Tout le monde, même les plus grands adversaires du sulpicien, le louèrent de son dévouement $^{13}$. Beaucoup plus tard, en 1840, un des successeurs de Mgr Panet, tout en se plaignant du procédé de $M$. Thavenet qui n'avait pas encore réglé ses comptes, se fit un devoir de lui exprimer "à différentes fois" sa reconnaissance pour les services rendus à ses prédécesseurs et à lui-même, ainsi qu'aux communautés du Canada ${ }^{14}$.

Le premier évêque de Montréal, Monseigneur Lartigue, Sulpicien, et quoique Canadien et peu suspect d'excessives sympathies françaises, ${ }^{15}$ résuma succintement les difficultés de $M$. Thavenet et de l'archevêché de Québec en reconnaissant que l'affaire des comptes était tellement difficile et compliquée que l'état de sa santé, singulièrement affectée depuis longtemps, ne lui permettait même pas d'y voir à fond. L'accusation contre Thavenet, affirma-t-il, n'avait pour cause que des "erreurs involontaires", et pas autre chose ${ }^{16}$.

\section{Origine des démêlés ecclésiastiques}

M. Thavenet se refusa à passer pour médiocre comptable. Malgré l'assurance répétée des partis adverses, il ne crut jamais que certains de leurs reproches ne visaient pas, d'une manière détournée, son intégrité personnelle et jusqu'à soupçonner en lui un agent frauduleux. Semblable réputation le déshonorait, pensait-il, ainsi que sa famille, le séminaire de Montréal dont il était membre et la Com-

13. Voir infra.

14. "Par là (en finissant les comptes) nous aurons tous deux fini nos affaires et vous aurez acquis un nouveau titre a la reconnaissance que je me suis fait un devoir de vous exprimer, à différentes fois, pour les services que vous avez rendus à mes prédécesseurs et à moi, ainsi qu'aux communautés de Canada." Discussion de $\mathbf{M}$. Thavenet... lettre de l'évêque de Québec à M. Thavenet, le 6 septembre 1841. L.S.

15. Il sera question de Mgr Lartigue au cours de cette étude.

16. Discussion de M. Thavenet... lettre de Mgr Lartigue a $M$. Thavenet, le 25 janvier 1840. L.S. 
pagnie de Saint-Sulpice à laquelle il avait l'honneur d'appartenir ${ }^{17}$. Avec son esprit un peu querelleur et méfiant, il en vint à juger qu'en cette affaire, il n'était pas seul en cause.

Pour tout dire il se persuada que l'Évêque de Québec et les communautés de cette ville lui en voulaient parce qu'il était Sulpicien et parce qu'il avait personnellement beaucoup contribué à renverser les plans de l'évêque de Québec qui aurait cherché à diminuer, voire à supprimer, l'institut sulpicien de Montréal et à chasser les Français du Canada ${ }^{18}$. Ainsi, M. Thavenet se plaçait lui-même au centre de cette querelle ecclésiastique entre Français et Canadiens. Il se croyait et se disait la victime choisie par l'Évêque de Québec pour se venger de toute la communauté de Saint-Sulpice et pour donner libre cours à sa xénophobie.

M. Thavenet avait certainement raison de croire à quelque sentiment anti-français dans les milieux religieux du Canada. Il avait tort de penser que le refus de Mgr Panet et de ses successeurs d'accepter sa reddition de comptes provenait uniquement de là. L'abbé, nous l'avons dit, n'avait rien d'un bon comptable ${ }^{19}$. L'on ne saurait davantage soutenir qu'en sa méfiance ou en ses critiques de la gestion de $M$. Thavenet, l'Évêque de Québec ait cédé à un simple sentiment d'animosité. Tout au plus peut-on se demander si ses critiques eussent fait voir autant de sévérité, si l'abbé n'avait pas été un Sulpicien.

Les discussions entre l'évêché de Québec et $M$. Thavenet s'élevèrent à un ton tellement acerbe qu'à vrai dire elles n'aboutirent à nulle solution. Un très grand nombre de personnalités eurent beau s'interposer comme médiateurs; l'affaire fut portée à la Sacrée Congrégation de la Propagande. Nous reviendrons, à la fin de cette étude, sur la suite de cette querelle entre $\mathrm{M}$. Thavenet et $\mathrm{M}$. Maguire. Pour le moment quittons l'affaire des comptes de Monsieur Thavenet, de fort relative importance. Étudions plutôt les événements qui contribuèrent à opposer les Canadiens aux Français

17. Ibid., Mon affaire avec Monseigneur..., 6.

18. Infra.

19. Nous avons examiné attentivement l'amas de registres qui constituent les comptes de $M$. Thavenet ainsi que les lettres et mémoires qu'il écrivit pour se justifier des reproches qu'on lui faisait. Il est indiscutable que $M$. Thavenet fournissait des pièces qui n'étaient ni très claires ni très précises. 
dans le Bas-Canada. Pour quelles causes les évêques et le clergé de ce pays se seraient-ils attaqués à la communauté même de SaintSulpice, à laquelle selon les dires de $M$. Thavenet, on cherchait précisément à créer des difficultés?

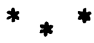

La mésentente entre Français et Canadiens, c'est-à-dire, entre les Sulpiciens et les évêques et le clergé du Canada, provenait de trois sources: on crut que le diocèse de Québec voulait saisir les biens du Séminaire de Montréal qui étaient considérables; on crut encore que les évêques de Québec tentaient d'empêcher l'entrée de Sulpiciens français dans leur maison de Montréal afin de laisser la place aux Sulpiciens canadiens; on crut enfin que ces mêmes évêques faisaient leur possible pour enlever la cure de Montréal aux Messieurs du séminaire. Entre chacune de ces accusations il y avait d'ailleurs interdépendance. A cela ajoutons des divergences de vue sur les candidats à présenter pour le siège de Québec et, par la suite, pour celui de Montréal, une fois ce second siège établi.

Pendant tout le XVIIe et XVIIIe siècles, la communauté de Saint-Sulpice avait été très bien vue et appréciée en Nouvelle-France. Ses premiers prêtres débarquèrent à Montréal en 1657 et, malgré quelques difficultés d'abord avec les Jésuites, puis avec Mgr François de Montmorency-Laval, arrivé au Canada en 1659 comme Vicaire Apostolique ${ }^{20}$, ils se rendirent rapidement indispensables à la colonie ${ }^{21}$. En 1663 ils devinrent les seigneurs de l'Ile de Montréal;

20. M. Gabriel de Queylus, premier supérieur de Saint-Sulpice en Amérique Française, se heurta au supérieur Jésuite, dès son arrivée au Canada, sur l'exercice de ses pouvoirs de grand Vicaire de l'Archevêque de Rouen. Plus tard, Mgr de Laval accusera les Sulpiciens d'élever autel contre autel et de troubler la paix religieuse du pays par leurs machinations, fraudes et insinuations sournoises. Sur les débuts de la congrégation de Saint-Sulpice et ses difficultés avec les Jésuites et le Vicaire Apostolique, voir Paradis, l'Influence de l'Archevêque de Rouen en Nouvelle-France (16401674).

21. Sur la grande ceuvre de Saint-Sulpice au Canada voir, par exemple, Henri Gauthier, La Compagnie de Saint-Sulpice au Canada (1912); du même auteur "SaintSulpice au Canada", La Revue Canadienne (mars 1912), 193-210; les ouvrages de Olivier Maurault, surtout Marges d'histoire (1930); et E. Levesque, L'Oeuvre de la Compagnie St Sulpice dans l'Amerique du Nord, Canada et Etats-Unis (1929). 
plus tard ils avaient ajouté à leurs biens les seigneuries du lac des Deux-Montagnes, puis, en 1735, la moitié des seigneuries de Bourchemin et de Saint-Herman, cette dernière sur la rivière Yamaska. Ces deux dernières furent vendues en $1796^{22}$.

Outre la considération que leur valaient ces richesses, les Sulpiciens eurent l'appui et le concours de tous les évêques français, au nombre de sept, qui succédèrent à $\mathrm{Mgr}$ de Laval sur le trône de Québec ${ }^{23}$. De fait, les Sulpiciens furent assez puissants pour que le supérieur général ait pu faire nommer un ancien missionnaire de Montréal comme auxiliaire de l'Évêque de Québec et, par la suite, lui assurer la succession sur le trône épiscopal, en septembre $1733^{24}$. Ce prélat, Mgr Pierre-Herman Dosquet, remit l'Église du Canada sous l'influence de ses anciens confrères en choisissant deux Sulpiciens comme vicaires généraux et un ancien Sulpicien comme doyen du chapitre. Ce Flamand naturalisé Français voulut ensuite réunir les deux séminaires de Montréal et de Québec au détriment de ce dernier. Et, avant de repartir pour la France, c'est lui qui fit don des seigneuries de Bourchemin et St-Herman au Séminaire de Montréal ${ }^{25}$. Ainsi les Messieurs de Saint-Sulpice pouvaient faire ce qu'ils voulaient au Canada; il en fut ainsi pendant le reste du régime français. Mgr de Pontbriand, le dernier évêque de Québec pendant cette période, avait reçu sa formation cléricale chez eux et c'est Saint-Sulpice qui le fit nommer évêque ${ }^{26}$. Après la chute de Québec, Mgr de Pontbriand se réfugia dans la maison des Sulpiciens de Montréal et e'est de là qu'il dirigea son diocèse, du ler octobre 1759 jusqu'à sa mort, le 8 juin 1760. Par testament il légua ses livres, ses

22. H. Gauthier, Sulpitiana (Montréal, 1926), 171.

23. M. Thavenet dira de ces sept évêques français: “...Tous n'ont donné aux prêtres de Saint-Sulpice que des marques de confiance, d'estime, d'affection, de bonté et de reconnaissance." Discussion sur la cure de Montréal (1830-31). Sagra Congregazione de Propaganda Fide ponente l'Eme, et Rmo Sig. Cardinale D. Placido Zurla Ristretto con sommario sulla instanza dei Sacerdoti Sulpiciani del Seminario di Montréal nel Basso Canada, accio l'Arcevescovo di Quebec non impedisca l'ammissione di Sacerdoti Sulpiciani Francesci nel Seminario medessimo (Aprile), Anno MDCCC$X X X I$, p. 6 du sommario).

24. Maurault, Nos Messieurs (1936), 72-74.

25. Ibidem.

26. Maurault, Le vieux séminaire de Notre-Dame de Montrél, (1925), 17-18. 
meubles, argenterie et espèces à la Congrégation, en guise de reconnaissance et d'attachement ${ }^{27}$.

L'attachement du clergé et de l'épiscopat du Canada à SaintSulpice semble avoir continué après la capitulation de la ville de Montréal et la cession de la colonie à l'Angleterre. Monsieur Étienne Montgolfier, Supérieur des Sulpiciens au Canada, fut choisi par le chapitre de Québec pour succéder à Mgr de Pontbriand en 1763. Ce ne furent pas les Canadiens qui entravèrent la grande influence des ecclésiastiques de Montréal, mais bien le gouvernement anglais, lorsqu'il refusa M. Montgolfier comme évêque de Québec ${ }^{28}$. Ce gouvernement porta un autre grand coup à la Compagnie en la menaçant dans ses biens. Tous les Français se sont vus soumis à une rigoureuse alternative: ou devenir citoyens britanniques et demeurer à ce titre dans la colonie, ou s'en retirer et se défaire dans un délai de dix-huit mois des biens qu'ils y possédaient ${ }^{29}$.

Le Séminaire de Saint-Sulpice de Paris eut done à résoudre un véritable dilemme. Il ne pouvait plus rester en possession de ses seigneuries au Canada. En toute justice il aurait pu les vendre et il semble que le gouvernement ait voulu les acheter. Plutôt que de se défaire de ses biens, le Séminaire de Paris décida d'en faire une cession gratuite à tous les membres de la Communauté qui consentiraient à devenir sujets anglais pour continuer sur place le ministère. Cet acte fut signé a Paris le 31 janvier 1765 et enregistré a Québec le 11 juin de la même année. Sur les quarante sulpiciens en Nouvelle-France, vingt-huit acceptèrent cette expatriation et prêtèrent serment de fidélité au roi d'Angleterre ${ }^{30}$.

Ainsi l'œuvre des Sulpiciens français aurait pu se continuer au Canada au prix de leur citoyenneté. Mais l'Angleterre para aussi à ce danger. Le même acte qui interdisait la possession de terres an-

\section{Ibidem.}

28. Le gouvernement anglais consentit à son élévation a l'épiscopat mais sans lui accorder aucun signe extérieur de sa dignité et pourvu qu'il format son chapitre des membres de sa communauté. Le gouverneur de Québec, qui avait aussi à donner son consentement, le refusa. M. Montgolfier sur le désir de Paris ne fit aucune opposition. Gauthier, La Compagnie..., 235.

29. Ibid., Maurault, Marges d'Histoire, 15.

30. Ibidem. 
glaises par des étrangers, fit défense aux ordres religieux de se recruter en France ${ }^{31}$. La congrégation de Saint-Sulpice et les autres communautés religieuses avaient donc le choix de devenir canadiennes ou de s'éteindre complètement.

Le sentiment anti-français des autorités britanniques ne laissa pas de se communiquer au clergé canadien. Le prêtre du pays s'habitua à faire une distinction qu'il faisait déjà avant 1760 , entre l'ecclésiastique de France et lui-même. Il ne s'ensuit pas que le Canadien approuvât nécessairement toute l'administration anglaise de son pays. D'autre part le Français né en France paraît avoir été beaucoup plus docile et favorable au pouvoir établi ${ }^{32}$. Mais, par sa propagande au temps de la Révolution et des guerres napoléonniennes, le gouvernement anglais réussit tout de même à séparer le Canadien de la France. Fait évident à qui observe ce qui se passa au Canada de 1763 au début du siècle suivant.

La fissure qui devait se produire entre Français et Canadiens n'apparut pas immédiatement. Malgré l'ostracisme dont le gouvernement frappait les prêtres étrangers, les Messieurs de Saint-Sulpice se sentirent assez forts et assez nombreux pour se charger de deux œuvres nouvelles: la fondation en 1767 d'un petit séminaire et, six ans plus tard, du premier collège de Montréal ${ }^{33}$.

Activité et puissance qui seraient bien incapables de se prolonger. Les années allaient réduire le nombre des membres de la communauté sulpicienne, surtout l'élément venu de France. En dépit d'instances répétées auprès de Londres et auprès de tous les amis qui auraient pu les aider, les Messieurs de Montréal ne purent se faire remplacer par des Sulpiciens français. Les Anglais forcèrent à se rembarquer les quelques religieux qui essayèrent d'entrer en fraude à Montréal ${ }^{34}$. En 1783, faute de recrutement, 75 paroisses man-

31. Maurault, Marges d'Histoire, 15.

32. Voir infra pour les marques de fidélité des Sulpiciens français envers la couronne d'Angleterre, même au moment où les Canadiens français se révoltaient contre le gouvernement anglais.

33. E. Levesque, L'œuvre de la Compagnie..., 12; Gauthier, La Compagnie de Saint-Sulpice..., 114.

34. Au moins trois Sulpiciens essayèrent de s'introduire à Montréal a l'Italienne, c'est-d-dire, incognito. Ils furent tous découverts par les Anglais et exclus du Canada. Maurault, Nos Messieurs, 29. 
quaient déjà de curés ${ }^{35}$. Dix ans plus tard il ne restait que trois Sulpiciens français dans tout le Canada: M. de Montgolfier qui dut résigner ses fonctions de supérieur à cause de ses infirmites, et $M$. Gabriel Brassier son remplaçant, lui-même épuisé et paralytique. Pour seconder ces vieillards, cinq prêtres dont un seul Français, M. Poncin, font avec eux, toute la Congrégation ${ }^{36}$.

D'après $M$. Thavenet, certains prêtres canadiens voyaient avec joie cette extinction du personnel français de la maison de Montréal. Un certain ressentiment se développait, disait-on, contre le clergé français. On en aurait souhaité la disparition pour entrer en possession de ses biens ${ }^{37}$.

Si les Canadiens avaient vraiment souhaité l'extinction complète des Sulpiciens français, la Révolution française leur enleva cet espoir. Nombre d'ecclésiastiques français avaient émigré à Londres. Le gouvernement britannique crut que ces proscrits pourrajent être à l'étranger d'excellents propagandistes contre leur pays. En septembre 1794, onze Sulpiciens réfugiés en Espagne purent gagner l'Amérique et le gouverneur de la colonie ferma les yeux sur l'arrivée de ces émigrés ${ }^{38}$. Vers 1800 une vingtaire de Sulpiciens français résidaient de nouveau au Canada ${ }^{39}$. La communauté sulpicienne redevenait francisée.

Jusqu'alors l'épiscopat de Québec, devenu britannique à la conquête, s'était montré assez favorable à Saint-Sulpice et aux Français. Les deux premiers évêques, Mgr Briand et Mgr Hubert, sont réputés amis de ce groupe ${ }^{40}$. Mais le troisième, Mgr Denaut, aurait fait preuve d'une tout autre mentalité. Toujours d'après l'abbé Thavenet, "il disait librement qu'il ne voulait plus de Français" pour

35. Gauthier, La Compagnie de Saint-Sulpice... 235.

36. Discussion sur la cure... Sagra Congregazione... (A prile) Anno MDCCCXXXI $8 \mathrm{du}$ sommario; E. Levesque, L'ceuvre de la Compagnie... 13.

37. Cette accusation revient souvent dans les écrits de M. Thavenet. Par exemple, voir Discussion sur la cure de Montréal... Sagra Congregatione... (A prile) Anno $M D C C C X X X I$, p. 8 du sommario.

38. Maurault, Nos Messieurs, 29; E. Levesque, L'œuvre de la Compagnie..., 114. M. Thavenet était un de ces onze.

39. Discussion sur la cure de Montréal... Sagra Congregazione... (A prile) Anno $M D C C C X X X I, 9$ du sommario.

40. Ibid., 8-9 du sommario. 
être les maîtres et seigneurs des Canadiens. En d'autres termes, il aurait cherché à s'émanciper de la tutelle des étrangers ${ }^{41}$. En sympathie avec ceux qui disaient du mal des Messieurs, Mgr Denaut serait allé jusqu'à ajouter ses railleries aux propos de ses prêtres. Les Sulpiciens lui auraient même imputé le nouvel ostracisme dont le gouvernement anglais frappa l'émigration de prêtres français dans la colonie. Et le gouverneur de la colonie aurait été heureux de porter un coup à la France, tout en faisant plaisir aux ecclésiastiques du Canada ${ }^{42}$.

Incapable de se recruter outre-mer, et voyant leur œuvre en danger de périr, les Messieurs de Saint-Sulpice se sentirent forcés de combler leurs vides avec des sujets du pays. Mgr Denaut s'en montra très heureux et donna volontiers la permission à quelquesuns de ses prêtres d'entrer dans la congrégation. C'est à ce moment que le Séminaire de Montréal accepta l'abbé Lartigue, qui en devenant successivement évêque auxiliaire de Québec et premier évêque de Montréal, occasionnerait de si douloureux démêlés ${ }^{43}$.

Pour l'abbé Thavenet, l'admission de l'abbé Lartigue, c'était pour les évêques de Québec, une tête de pont dans la maison de SaintSulpice. Ils n'en restèrent pas là. Estimant nécessaire leur mainmise sur cette institution ils se seraient ingéniés à machiner des plans pour arracher aux Sulpiciens et la propriété de leurs grandes seigneuries et leur influence sur les Canadiens de leur district. Au début du XIXe siècle, Mgr Plessis, évêque de Québec, continue la politique de son prédécesseur Mgr Denaut. Il laisse entrer de ses prêtres à Saint-Sulpice et essaie même d'introduire son coadjuteur parmi eux. D'après M. Thavenet qui fournit cette explication à Rome, il raisonne comme suit: si le coadjuteur est admis dans la communauté et en devient membre, de ce fait, il faudra, vu sa dignité, le nommer supérieur; une fois supérieur, il se gardera de faire venir de France des prêtres de Saint-Sulpice, mais à mesure que les vieux Français disparaitront, il les remplacera par des Canadiens. Ainsi la communauté sera bientôt complètement libérée des étrangers.

\section{Ibidem. \\ 42. Ibidem.}

43. Ibid., 10 du sommario. 
Les Sulpiciens qui auraient vu clair dans le dessein de Mgr Plessis, s'excusèrent de ne pas recevoir le coadjuteur dans leur maison ${ }^{44}$.

Sur ce, l'évêque de Québec ne permit plus à aucun prêtre du diocèse de s'agréger à la Sociétét ${ }^{45}$. C'était changer de tactique. Il semble qu'il ait voulu tout simplement laisser mourir le Séminaire d'épuisement. Avait-il pour autant perdu l'espoir de faire passer le contrôle de la maison et de ses multiples dépendances entre des mains canadiennes? Depuis l'introduction de Saint-Sulpice en Amérique, aucun sujet né au Canada n'en avait été le supérieur; il semble même que les religieux indigènes n'y aient jamais occupé des postes très importants, sauf durant les années où il n'y avait pas assez de Français pour les assumer tous ${ }^{46}$. En 1829, ces Messieurs préféreront, à tous les Canadiens, un ancien ministre protestant, citoyen des États-Unis, pour remplir les fonctions curiales de la paroisse de Montréal ${ }^{47}$. En plus de la méfiance à l'égard des prêtres du pays, il semble qu'ils ne les aient pas toujours trouvés à la hauteur du ministère qu'ils auraient eu à leur confier ${ }^{48}$. De leur côté les Canadiens ne trouvaient pas moins à redire de la science de plusieurs de leurs confrères venus de France ${ }^{49}$.

\section{Le grand vicaire Lartigue}

Le premier incident qui, dans la suite, allait mettre en conflit Mgr Plessis et Saint-Sulpice, se produit une douzaine d'années

44. Ibid., 10-11 du sommario.

45. Ibid., 11.

46. Même en 1794, quand il n'y avait que trois Français dans la maison, M. Montgolfier, le supérieur, fut remplacé par un autre Français, M. Brassier, bien que celui-ci fât paralysé. Voir supra.

\section{Infra.}

48. C'est à cause de ceci que Saint-Sulpice refusa deux candidats canadiens en 1830. Les Messieurs disaient qu'ils ne jugeaient pas les hommes "par terre de leur naissance" mais par leur valeur. Discussion sur la cure de Montréal, 1830-1831. Lettre du Séminaire de Montréal à l'évêque de Québec, le 2 aout 1830. Voir infra pour les troubles qui suivirent cette décision de Saint-Sulpice.

49. Mgr Panet, l'évêque de Québec disait de M. Fay, un Sulpicien, qu'il avait fait de minces études et qu'en plus il était faible de caractère. Discussion sur lo cure... Sagra Congregazione... MDCCCXXXI, 62. 
après l'échec de la tentative pour introduire un évêque dans le séminaire de Montréal. Cette fois, selon encore l'interprétation sulpicienne, le dessein de Mgr Plessis est tout simple: se choisir un collaborateur parmi les membres canadiens de la communaute; et, de cette façon, pénétrer dans la maison sans qu'on puisse l'accuser d'y introduire des étrangers ${ }^{50}$. A vrai dire, les habitants de Montréal désiraient depuis très longtemps un évêché en titre ${ }^{51}$ et il est probable que l'Évêque de Québec voulait la même chose. Mgr Plessis ne s'opposait pas au démembrement de son diocèse. En 1816, Rome lui avait enlevé la Nouvelle-Écosse qui fut confiée a un Vicaire Apostolique; d'autres subdivisions avaient suivi cellelà ${ }^{52}$. En 1819 l'évêque résolut même d'aller en personne à Rome demander l'érection de deux autres diocèses, ceux de Montréal et de la Rivière-Rouge.

Sur le navire il voyagea avec l'abbé Jean-Jacques Lartigue qui s'en allait à Londres plaider la cause du Séminaire de Montréal, menacé par les Anglais, de perdre ses biens ${ }^{53}$. Est-ce le hasard qui avait amené Mgr Plessis et ce Sulpicien canadien sur le même bateau ? M. Thavenet et plusieurs de ses confrères ont accusé l'évêque d'avoir provoqué l'envoi de M. Lartigue à Londres dans l'intention préméditée de le soustraire à l'influence de son supérieur et afin de lui proposer l'évêché de Montréal dans un lieu où il lui serait impossible de prendre l'avis de ses confrères sulpiciens ${ }^{54}$. Monseigneur Plessis quitta M. Lartigue à Londres. Il vit le secrétaire des colonies, Lord Bathurst, puis se rendit à Rome. Il ne put obtenir l'érection d'un évêché à Montréal. On ne lui accorda qu'un grand vicaire revêtu du caractère épiscopal. Ainsi en décida Lord Bathurst, a l'encontre des intentions de l'évêque de Québec et du Saint Siège ${ }^{\text {ts }}$.

50. Discussion sur la cure de Montreal... Sagra Congregazione... (Aprile) Anno $M D C C C X X X I, 11$ et suiv.

51. C'est Londres qui s'opposa toujours aux démarches des évêques de Québec pour obtenir cette érection. Maurault, Nos Messieurs, 77-78.

52. Ibidem, 80.

53. Ibidem. Voir infra pour la question des biens de Saint-Sulpice.

54. Discussion sur la cure de Montréal... Sagra Congregazione... (Aprile) $M D C C C X X X I, 11$ et suiv.

55. Maurault, Nos Messieurs, 80 et suiv. 
Entre temps M. Lartigue reçoit du supérieur général de Saint-Sulpice la permission d'accepter le poste qu'on lui proposait. Il est entendu pourtant que, tout en restant sulpicien, il cessera de cohabiter avec ses confrères ${ }^{56}$.

Après son sacre en l'église Notre-Dame de Montréal le 21 janvier 1821, Mgr Lartigue s'installe tout de même parmi ses anciens confrères et fait ériger son trône épiscopal dans leur église paroissiale. Trois semaines après la cérémonie, les Sulpiciens, profitant d'une absence de l'évêque, déménagent ses meubles du Séminaire à l'HôtelDieu qui était voisin, et, au mois de juin, ils enlèvent son trône de l'église Notre-Dame ${ }^{57}$. Ainsi Mgr Lartigue et Mgr Plessis sont bien avertis que les Messieurs n'abandonneront pas facilement leurs droits à l'autonomie dans leurs seigneuries et que l'élément français n'est pas prêt à céder la place aux sujets canadiens. De plus, il leur est signifié que Mgr Lartigue a dépassé ses droits de grand vicaire en voulant se faire honorer comme évêque en titre. L'évêque de Boston, Mgr Cheverus, partage sur cette question le point de vue des Sulpiciens ${ }^{58 \mathrm{a}}$.

Les procédés des prêtres du Séminaire blessèrent profondement Mgr Lartigue. Comme nous l'avons dit, ce prélat était Canadien et avait été prêtre du diocèse de Québec avant d'entrer à St-Sulpice, avec la permission de Mgr Denaut. Partageait-il les sentiments des évêques de Québec envers l'élément français, même avant son sacre comme évêque de Telmesse? On peut le présumer. Son expulsion et du séminaire et de l'Église de Montréal, n'arrangea pas les choses. Ses difficultés avec les fabriciens de l'Église sulpicienne de Montréal, le jour où il voulut ériger une cathédrale et un palais épiscopal dans

56. Ibidem.

57. Ibidem., 81-83.

58a. "Je vous avoue que $M$. Roux et ses saints confrères m'ont inspiré un tel respect que je crois leur influence favorable à la religion, et je suis persuadé qu'ils désirent voir l'épiscopat très respecté. Il m'a paru que la seule question était de savoir si un évêque qui, au caractère près, n'est que votre grand vicaire, avait tous les droits et les honneurs d'un évêque diocésain et quand ils seraient dus, serait-ce dans une église qui depuis 180 ans est a Saint-Sulpice qu'il devrait désirer d'en jouir constamment?"' Mgr Cheverus, évêque de Boston à Mgr Plessis, évêque de Québec, le 12 octobre 1821, Maurault, Nos messieurs, 85-86. 
cette ville ne firent qu'aggraver la situation ${ }^{55^{b}}$. La correspondance de Mgr Lartigue, surtout à partir de 1830, est remplie de doléances contre les Sulpiciens qui veulent "surcharger la maison de Montréal de nouveaux étrangers sans la permission de l'évêque" alors qu'ils refusent de bons sujets canadiens ${ }^{59}$. Il est surtout froissé de voir ses compatriotes demeurer indéfiniment les serviteurs des Français "qui gouvernent tout, en dehors et en dedans, indépendamment des évêques" ${ }^{\prime}$. Aussi, surveille-t-il de très près l'introduction d'étrangers dans les maisons sulpiciennes de Montréal. Et il ne se fait pas faute d'en référer fidèlement à l'Évêque de Québec, surtout après que celui-ci eât interdit telle agrégation à la communauté sulpicienne ${ }^{61}$. En 1830, il conseille à Mgr Panet, successeur de Mgr Plessis à Québec, de ne pas nommer M. Quiblier son grand vicaire, parce que "c'est encore un français non naturalisé" ${ }^{62}$. Et il écrit au supérieur du collège anglais à Rome que "ce jeune sulpicien, professeur au collège, sans connaissance du ministère, a été élu supérieur du Séminaire de Montréal, contre le désir général du clergé et du peuple de ce pays" ${ }^{63}$.

58b. La construction de la cathédrale et de l'évêché fut entreprise à peu près au même moment que les fabriciens de Notre-Dame de Montréal commencèrent la construction d'une nouvelle église.

59. Fonds canadien... lettre de Mgr Lartigue a l'abbé Wiseman, recteur du Collège Anglais à Rome, le 7 Mai 1831. Voir aussi les lettres de celui-ci à Monsieur D.-B. Viger a Rome, le 9 juillet 1831: "M. Thavenet est a Rome, non pour rendre service a notre diocèse, mais pour obtenir du Saint-Siège de venir en Canada avec une troupe d'autres prêtres français inonder le séminaire de Montréal"; a Mgr Panet, le 7 Janvier 1832; à Monsieur D.-B. Viger, le 10 janvier 1832: il s'oppose à l'entrée de nouveaux Sulpiciens en Canada "car notre pays peut alimenter suffisamment ce séminaire et je ne verrais pas en général d'un bon œil venir d'Europe ici des maítres pour nous régir, ce qui est pourtant un plan assez à la mode parmi nos canadiens d'aujourd'hui"; et cotera.

60. Ibidem, lettre de Mgr Lartigue à l'évêque de Fussada, le 31 Décembre 1832: "...les Canadiens ne seront que les très humbles serviteurs de ces étrangers qui gouvernent tout, en dedans et en dehors, indépendamment des évêques".

61. Ibidem, lettre de Mgr Lartigue à Mgr Signay, 1833, lui écrit que les Sulpiciens viennent de recevoir deux jeunes Français et Irlandais inconnus dans lour collège.

62. Ibidem, lettre de Mgr Lartigue à Mgr Panet, le 16 aout 1830; pour une autre réflexion anti-Quiblier voir, ibid., lettre au même, le 30 mai 1832.

63. Ibidem, lettre de Mgr Lartigue à l'abbé Wiseman à Rome, le 7 mai 1831. 
Vers la fin de sa vie, Mgr Plessis pose a Rome deux questions pertinentes au sujet des Sulpiciens. Rome ne répond point. Mgr Plessis ne pousise pas plus outre ${ }^{64}$. Son successeur, Mgr Panet, va reprendre l'offensive avec l'aide et l'encouragement de Mgr Lartigue. Le gouvernement anglais de la métropole et celui de la colonie se mélent de l'affaire, car il s'agit maintenant des biens du Séminaire de Montréal. C'est ici que $M$. Thavenet entre à nouveau en scène.

Wilfrid-H. Paradis, ptre

Docteur en droit canonique de l'Institut catholique de Paris, et docteur de l'Universite de Paris, Manchester, E.U.

(d suivre)

64. Ces questions étaient les suivantes: Un clerc ou un prêtre peut-il être agrégé a une communauté de prêtres séculiers, sans le consentement de l'évêque, à moins de produire un titre qui l'exempte de la demander ? Un évêque peut-il pour le plus grand bien de la religion tirer d'une communauté de prêtres séculiers un clerc ou un prêtre pour lui donner un emploi de plus grande importance même sano le consentement de la communauté ? Discuseion sur la cure de Montréal... Sagra Congregazione... (A prile) Anno MDCCCXXXI, 13 in sommario. Mgr Plessis ne réagit pas a l'arrivée de trois Sulpiciens de France entre 1823 et 1825. Ibid., 14. 